\title{
Strain rate behaviour of multi-phase and complex-phase steels for automotive applications
}

\author{
E. Cadoni ${ }^{1}$, N.K. Singh ${ }^{2}$, M.K. Singha ${ }^{2}$, and N.K. Gupta ${ }^{2}$ \\ ${ }^{1}$ DynaMat Laboratory, University of Applied Sciences of Southern Switzerland, 6952 Canobbio, Switzerland \\ 2 Department of Applied Mechanics, Indian Institute of Technology Delhi, New Delhi-110016, India
}

\begin{abstract}
A combined study on the mechanical behaviour of multi-phase 800 high yield strength steel (MP800HY) and complex-phase 800 steel (CP800) is carried out under tensile loads in the strain rate range from $0.001 \mathrm{~s}^{-1}$ to $750 \mathrm{~s}^{-1}$. Quasistatic $\left(0.001 \mathrm{~s}^{-1}\right)$ tests are performed on electromechanical machine, whereas, medium $\left(5 \mathrm{~s}^{-1}\right.$ and $\left.25 \mathrm{~s}^{-1}\right)$ and high strain rate $\left(250 \mathrm{~s}^{-1}, 500 \mathrm{~s}^{-1}\right.$ and $\left.750 \mathrm{~s}^{-1}\right)$ experiments are conducted on hydro-pneumatic machine (HPM) and modified Hopkinson bar (MHB) setup respectively. The thermal softening behaviors of the materials are investigated at quasi-static condition and the materials' $m$-parameters of the existing Johnson-Cook model are imposed in authors' previous work. Thereafter, the predicted flow stress by Johnson-Cook model has been compared with the experimental results.
\end{abstract}

\section{Introduction}

The requirement of lightweight and high strength materials in the automobile industry has motivated researchers to use different advanced high strength steels. Study of the mechanical behaviours of such materials at different loading rates is needed to design the auto-body structures for the maximum possible dissipation of crash energy. The advanced high strength steels can attain very high strengths while retaining moderate ductility, which makes them ideal for dissipating energy during an automotive crash [1-4]. Their inherent high strength allows structural components to be thinner, making the vehicle lighter and more fuel efficient. Multi-phase 800 High Yield strength steel (MP800HY) and complex-phase 800 steel (CP800) are two potential materials in the range of advanced high strength steels. These steels have good combination of strength and formability. These are used in automobile industries for high yield strength values especially in crash relevant parts such as side impact protection and bumpers, and also in structural parts including reinforcements or cross members. Singh et al. [5,6] studied the mechanical behavior of these steels at strain rates $0.001 \mathrm{~s}^{-1}$ to $750 \mathrm{~s}^{-1}$ under tensile loads. The authors' previous work is extended here to study the thermal softening behavior of the materials at quasi-static condition $\left(0.001 \mathrm{~s}^{-1}\right)$. The material $m$-parameter of the existing Johnson-Cook model has been imposed in the previous work and the predicted flow stresses by this model are compared with the experimental results at different strain rates. Thereafter, the overall mechanical characteristics are compared.

Quasi-static tests are performed on electromechanical machine at room temperature $\left(20^{\circ} \mathrm{C}\right)$ as well as high temperature $\left(200^{\circ} \mathrm{C}\right)$ to understand the high temperature effect in the deformation of the materials. The medium $\left(5 \mathrm{~s}^{-1}\right.$ and $\left.25 \mathrm{~s}^{-1}\right)$ and high strain rates $\left(250 \mathrm{~s}^{-1}, 500 \mathrm{~s}^{-1}\right.$ and $750 \mathrm{~s}^{-1}$ ) experiments are conducted on hydro-pneumatic machine (HPM) and modified Hopkinson bar (MHB) setup respectively. The descriptions of the used experimental techniques are discussed in authors' previous work $[5,6]$.

\section{Materials and specimen design}

The investigated materials, MP800HY and CP800 are obtained from FIAT research centre, Turin, Italy whose chemical composition in wt.\% is given in Ref. $[5,6]$. The microstructure of MP800HY consists of mainly ferrite matrix and granular bainite, whereas, the microstructure of CP800 contains small amounts of martensite, retained austenite and pearlite within the ferrite - bainite matrix where, Nital $\left(\mathrm{HNO}_{3}+\mathrm{CH}_{3} \mathrm{OH}\right)$ is used as the etching reagent. The fractographs of the materials tested at room temperature $\left(20^{\circ} \mathrm{C}\right)$ in quasi-static, medium and high strain rates are presented in Fig. 1. Fractographs of MP800HY is coarser, whereas, CP800 has smooth fracture. The cup like depressions (dimples) shows ductile fracture of the materials. The dimple increases and becomes deeper with increasing strain rate. These pictures are taken by SEM at the magnification of 2500x. Flat sheet specimens $[1,2]$ of the materials have gauge length $10 \mathrm{~mm}$, width $4 \mathrm{~mm}$ and, thickness $3 \mathrm{~mm}$ and $0.97 \mathrm{~mm}$ for MP800HY and CP800 respectively, are cut by electro discharging machine from the sheet materials following the direction of the lamination. The thin sheet metal specimen is inserted in the specimen holders by glue; subsequently, a steel dowel is used to prevent slippage. Both specimen and supports are threaded jointly for $8 \mathrm{~mm}$ for effective gripping with the bars.

\section{Results and discussion}

The experimental data of various experiments are investigated for the materials MP800HY and CP800 under tensile loads. The stress-strain curves of different strain rates at room temperature are compared and the measured experimental results are presented in $[5,6]$. Here, the quasi-static tests at high temperature $\left(200^{\circ} \mathrm{C}\right)$ are done in electromechanical machine (Fig. 2). The stressstrain curves (Figs. 3 and 4 ) of the materials in quasistatic condition are compared at room $\left(20^{\circ} \mathrm{C}\right)$ and high temperatures $\left(200^{\circ} \mathrm{C}\right)$. For $\mathrm{MP} 800 \mathrm{HY}$, the engineering 

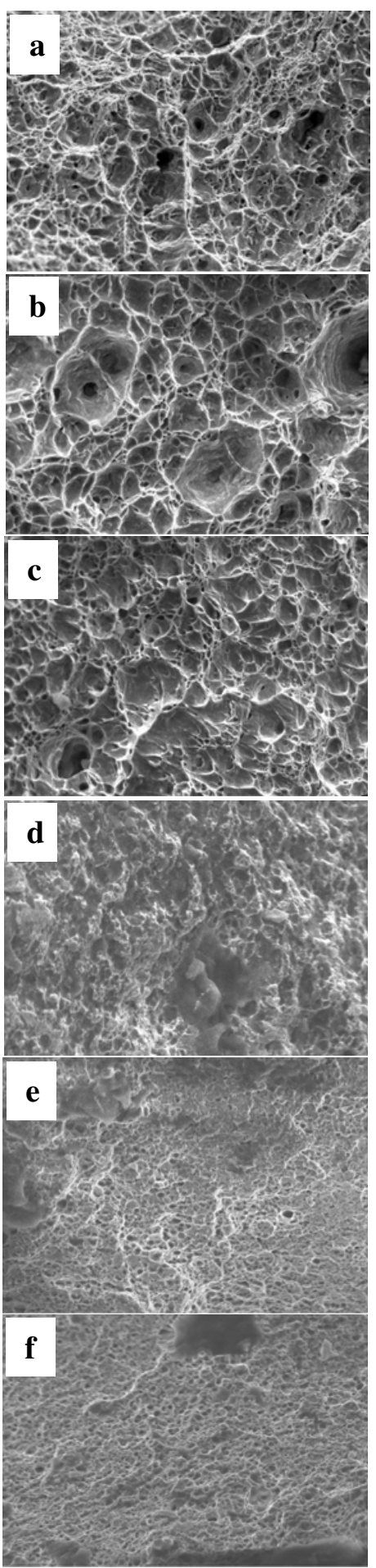

Fig. 1. Fractographs (2500x) of MP800HY [a. $0.001 \mathrm{~s}^{-1}$, b. $25 \mathrm{~s}^{-1}$, c. $750 \mathrm{~s}^{-1}$ ] and CP800 [d. $0.001 \mathrm{~s}^{-1}$, e. $25^{-1}$, f. $750 \mathrm{~s}^{-1}$ ] from SEM.

and true yield stresses are $782 \mathrm{MPa}$ and $785 \mathrm{MPa}$ respectively, whereas, the engineering and true ultimate tensile strengths are respectively $879 \mathrm{MPa}$ and $933 \mathrm{MPa}$ at $20^{\circ} \mathrm{C}$. At $200{ }^{\circ} \mathrm{C}, \mathrm{MP} 800 \mathrm{HY}$ has the engineering and true yield stresses $762 \mathrm{MPa}$ and $765 \mathrm{MPa}$ respectively, whereas, this material has engineering and true ultimate tensile strength respectively, $851 \mathrm{MPa}$ and $895 \mathrm{MPa}$. For

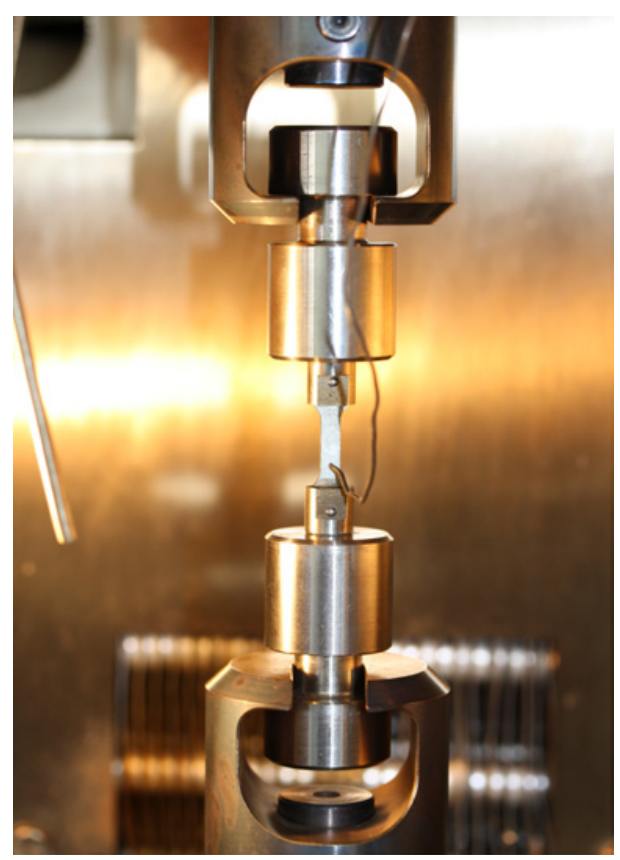

Fig. 2. Quasi-static tests at high temperatures on electromechanical machine.
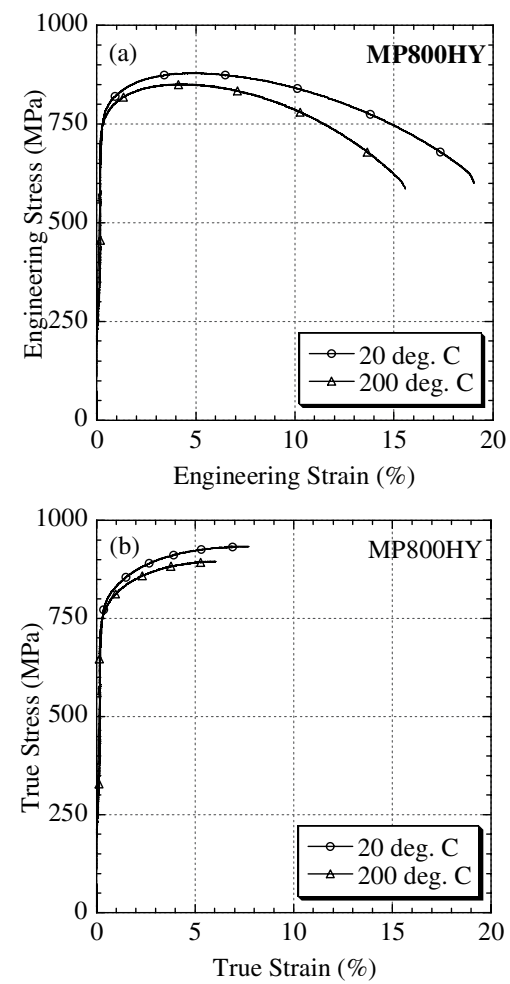

Fig. 3. Stress-strain curves of MP800HY (a) Engineering stressstrain (b) True stress-strain.

CP800, the engineering and true yield stresses are $685 \mathrm{MPa}$ and $689 \mathrm{MPa}$ respectively, whereas, the engineering and true ultimate tensile strength are respectively, $872 \mathrm{MPa}$ and $946 \mathrm{MPa}$ at room temperature. At $200^{\circ} \mathrm{C}, \mathrm{CP} 800$ has the engineering and true yield stresses $580 \mathrm{MPa}$ and $582 \mathrm{MPa}$ respectively, whereas, this material has the engineering and true ultimate tensile strengths respectively, 659 MPa and 

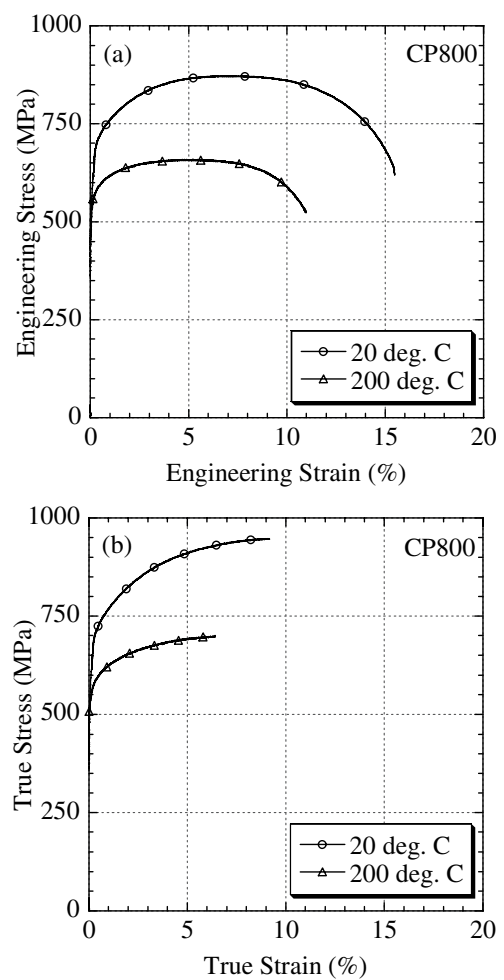

Fig. 4. Stress-strain curves of CP800 (a) Engineering stress-strain (b) True stress-strain.

$699 \mathrm{MPa}$. Thus, the flow stresses of both the materials decreases at $200{ }^{\circ} \mathrm{C}$. The variation of dynamic increase factor (DIF) with the obtained strain rate for yield stress and ultimate tensile strength are compared in Fig. 5. In case of MP800HY, DIF of U.T.S. slightly increases with increasing strain rate compared to that for Y.S., whereas, in case of CP800, DIF of U.T.S. decreases significantly with increasing strain rate at high strain rates in comparison to Y.S.

The Fig. 6 shows that reduction in area is more in MP800HY compared to CP800 at each strain rate. As the area under the stress-strain curve decreases at high temperature, the fracture energy and toughness of the materials will decrease. The elongation in the materials decreases, therefore, the ductility will decrease at the high temperature $\left(200^{\circ} \mathrm{C}\right)$.

\subsection{Material model}

Material model incarnates dynamic response of the material due to thermo-mechanical parameters and is the important basis for numerical simulation in metal plastic deformation process.

In order to have confidence in the results of such simulations, an accurate material model is required. Here, the material parameters of existing Johnson-Cook model are determined based on the experimental results and thereafter, the experimental flow stress is fitted with the predicted result. J-C model is purely empirical and is widely used model. These days, it is already in-built in most of the simulation software. This model can reproduce
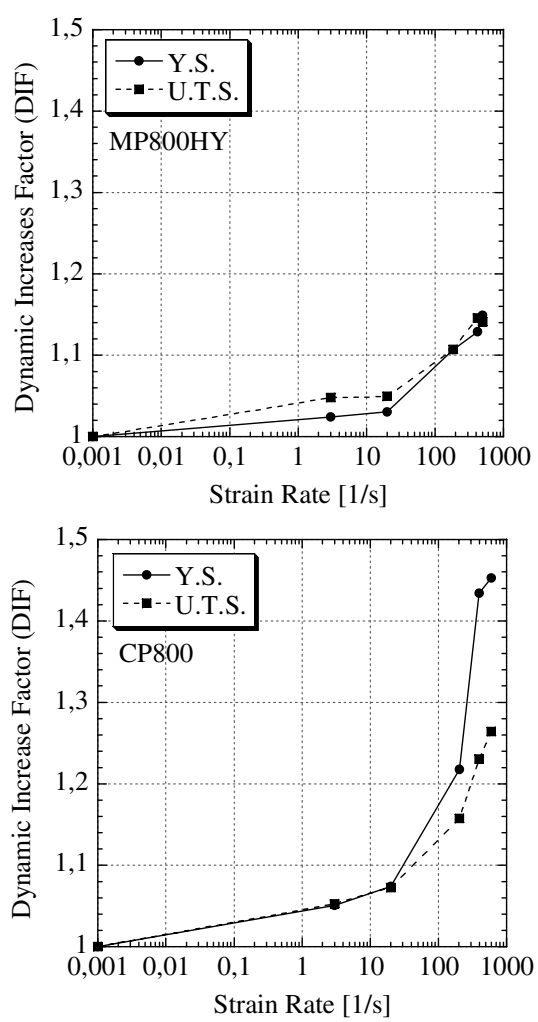

Fig. 5. Variation of dynamic Increase Factor with obtained strain rate for Y.S. and U.T.S.

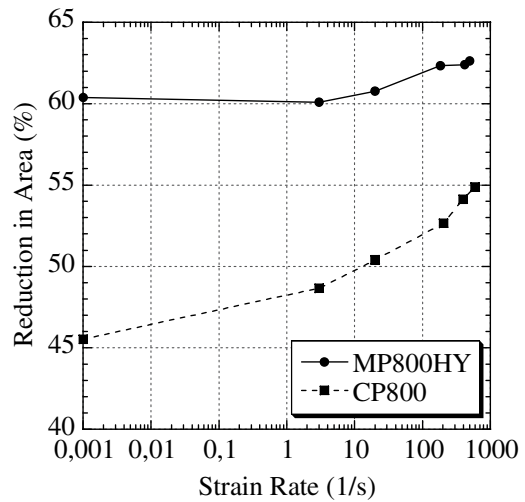

Fig. 6. Reduction in area at different obtained strain rates for MP800HY and CP800.

several important material responses observed in impact and penetration of metals.

The Johnson-Cook model is expressed [7] as

$$
\sigma=\left[A+B \varepsilon^{n}\right] \cdot\left[1+C \ln \varepsilon^{*}\right] \cdot\left[1-T^{*} m\right]
$$

Where, $\varepsilon$ is the equivalent plastic strain, $\dot{\varepsilon}$ is the strain rate, $\dot{\varepsilon}^{*}=\dot{\varepsilon} / \dot{\varepsilon}_{0}$ is the dimensionless plastic strain rate for $\dot{\varepsilon}_{0}=0.001 \mathrm{~s}^{-1}$

$$
\left(T^{*}=\frac{T-T_{R O O M}}{T_{M E L T}-T_{R O O M}}\right)
$$

$T^{*}$ is the homologous temperature, $A$ is the true yield stress, $B$ and $n$ represent the effects of strain hardening, $C$ is the strain rate sensitivity constant, $m$ is the thermal softening parameter. 

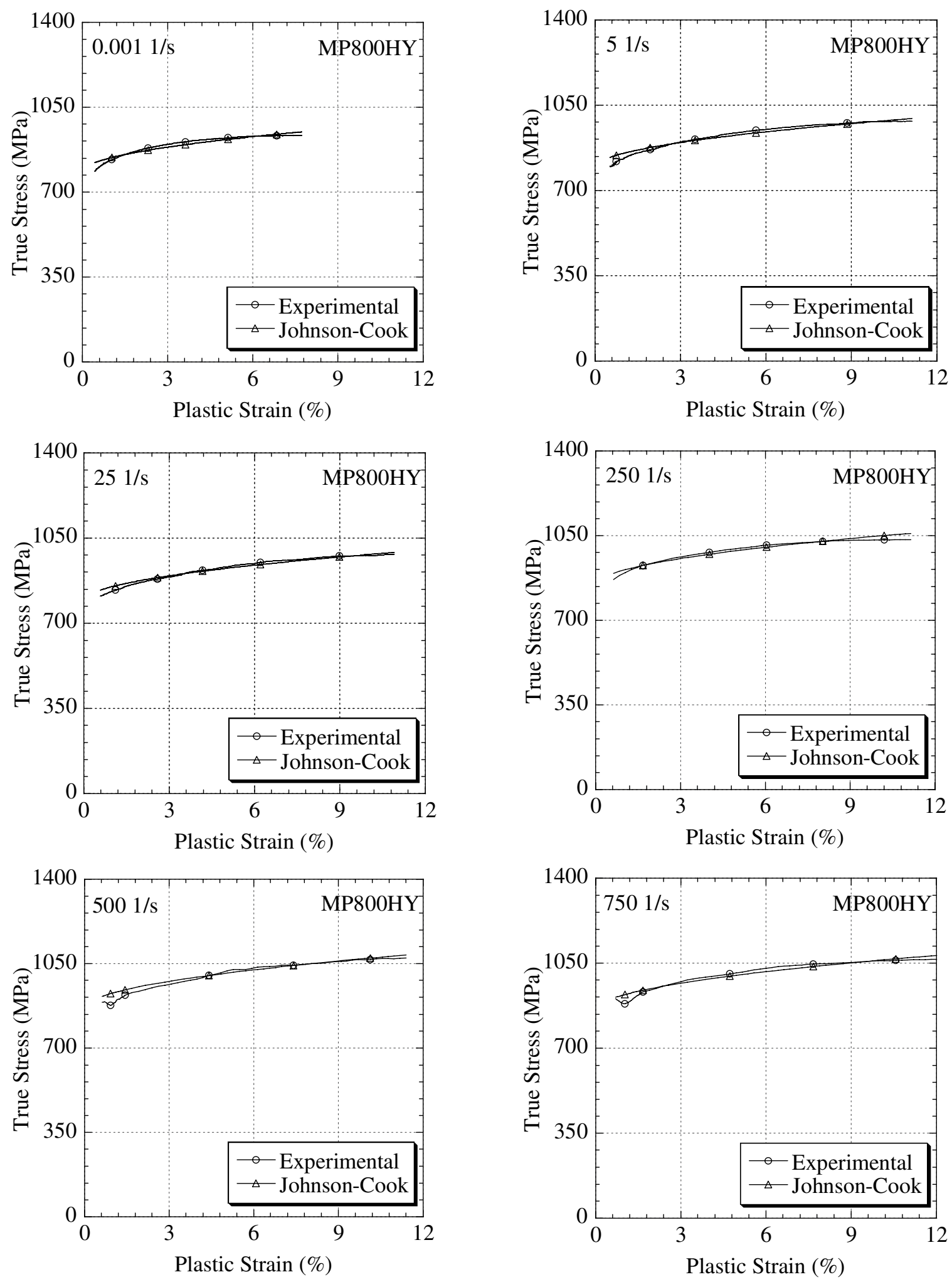

Fig. 7. Comparison of predicted flow stress of MP800HY by Johnson-Cook model with the experimental results.

The parameters $B$ and $n$ are determined by curve fitting method. The strain rate sensitivity parameter $C$ is obtained as the slope of the linear fit of natural logarithm of strain rate versus the ratio of dynamic yield stress to static yield stress. The parameter $m$ is defined as $\ln (1-R) / \ln \left(T^{*}\right)$ where, $R$ is the mean ratio of plastic stresses at high temperature and at room temperature. The estimated material parameters $(A, B, C, \mathrm{n}$ and $m)$ for the Johnson-Cook model are presented in Table 1 for both the steels. The predicted flow stresses are compared with the experimental results 

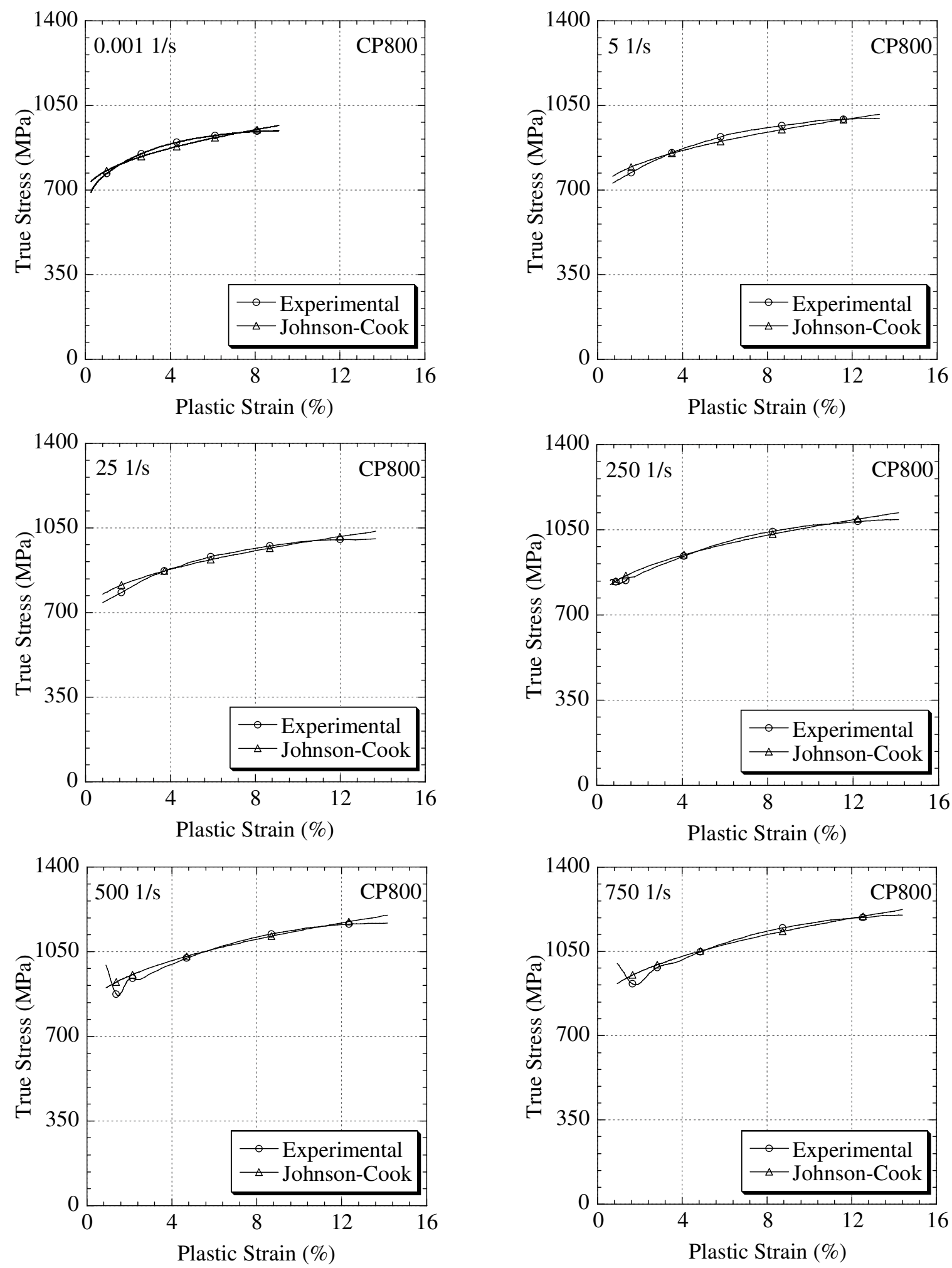

Fig. 8. Comparison of predicted flow stress of CP800 by Johnson-Cook model with the experimental results.

in Figs. 7 and 8 at different strain rates for MP800HY and CP800 respectively.

It is observed that the Johnson-Cook model with the estimated material parameters can represent the experimental data well and hence, can be recommended for the numerical simulation.

\section{Conclusions}

The investigated materials, MP800HY and CP800 are moderate strain rate sensitive. The flow stresses and ductility of the materials decrease at the high temperature, $200^{\circ} \mathrm{C}$ compared to that at room temperature, $20^{\circ} \mathrm{C}$ 
Table 1. Material parameters of Johnson-Cook model.

\begin{tabular}{|c|c|c|}
\hline Parameters & MP800HY & CP800 \\
\hline$A(\mathrm{MPa})$ & 785 & 689 \\
\hline$B(\mathrm{MPa})$ & 610 & 919 \\
\hline$n$ & 0.508 & 0.493 \\
\hline$C$ & 0.010769 & $0.01-0.015$ \\
\hline$m$ & $1.2-3.0$ & $1.2-2.8$ \\
\hline
\end{tabular}

Therefore, the fracture energy and toughness of the materials also decrease. Materials have ductile fracture. There is good agreement between the predicted results by Johnson-Cook model and the experimental results.

\section{Acknowledgements}

Authors thank Dr. Fabio D'Aiuto of FIAT Research Centre, Turin, Italy for providing the materials and D. Forni and M. Dotta of the DynaMat Laboratory, University of Applied Sciences of Southern Switzerland, for help in the experiments. The research was financially supported by the Indo-Swiss Joint Research Program, project IPP03 "Mechanical characterization of advanced materials under dynamic loads", sponsored by the "Department of Science and Technology" of India and "Swiss State Secretariat of Education and Research" of Switzerland.

\section{References}

1. M. Takahashi, A. Uenishi, H. Yoshida, H. Kuriyama. Advanced high strength steels for automobile body structures. Material Science Forum, 539-543, 43864390 (2007).

2. J. V. Slycken, P. Verleysen, J. Degrieck, J. Bouquerel, B. C. D. Cooman. Crashworthiness characterization and modeling of high-strength steels for automotive applications. Proc IMechE J Auto Eng, 220 (D), 391400 (2006).

3. V. Tarigopula, M. Langseth, O. S. Hopperstad, A. H. Clausen. Axial crushing of thin-walled high-strength steel sections. Int J Imp Eng, 32, 847-882 (2006).

4. N. Peixinho, N. Jones, A. Pinho. Experimental and numerical study in axial crushing of thin walled sections made of high-strength steels. J Phys IV France, 110, 717-722 (2003).

5. N. K. Singh, E. Cadoni, M. K. Singha, N. K. Gupta. Dynamic tensile behaviour of multi phase high yield strength steel. Materials and Design, 32, 10, 50915098 (2011).

6. N. K. Singh, E. Cadoni, M. K. Singha, N. K. Gupta. Quasi-static and dynamic tensile behavior of CP800 steel. Mechanics of Advanced Materials and Structures, (in press).

7. K. Vedantam, D. Bajaj, N. S. Brar and S. Hill. Johnson-Cook strength models for mild and DP590 steels. Shock Compression of Condensed Matter, CP845: 775-778, (2005). 